\title{
Relación entre depresión y promedio escolar en estudiantes de la Facultad de Psicología y Terapia de la Comunicación Humana, UJED, campus Durango
}

\section{Relation between depression and school average in students of the Faculty of Psychology and Therapy of Human Communication, UJED, Durango campus}

HERRERA-VARGAS, Isela Vanessa $\dagger^{1}$, RÍOS-VALLES, José Alejandro*1, 2, PÉREZ GONZÁLEZ, Ivonne $^{1}$ y VÁZQUEZ-RÍOS, Elda Raquel ${ }^{1}$

${ }^{1}$ Facultad de Psicología y Terapia de la Comunicación Humana, UJED.

${ }^{2}$ Instituto de Investigación Científica, UJED

ID $1^{\text {er }}$ Autor: Isela Vanessa, Herrera-Vargas / ORC ID: 0000-0002-9154-6978, Researcher ID Thomson: X-3314-2018, CVU CONACYT ID: 954357

ID $1^{\text {er }}$ Coautor: José Alejandro, Ríos-Valles/ ORC ID: 0000-0002-8407-3017, Researcher ID Thomson: X-3209-2018, CVU CONACYT ID: $313-266$

ID $2^{\text {do }}$ Coautor: Ivonne, Pérez-González/ ORC ID: 0000-0002-5010-230X, Researcher ID Thomson: X-8722-2018, CVU CONACYT ID: 954229

ID $3{ }^{\text {er }}$ Coautor: Elda Raquel, Vázquez-Ríos/ ORC ID: 0000-0003-1084-6053, Researcher ID Thomson: X-9849-2018, CVU CONACYT ID: 888617

\section{Resumen}

Objetivo: identificar la relación entre la depresión y el promedio escolar en estudiantes de la Facultad de Psicología y Terapia de la Comunicación Humana, UJED. La depresión se evaluó mediante el Test de Beck a 110 alumnos. Es un estudio cuantitativo, exploratorio, no experimental, observacional y transversal, con análisis estadístico descriptivo y correlacional, con muestreo no probabilístico por conveniencia. El alfa de Chronbach de los datos analizados fue de 0.89 . El 50\% de los estudiantes presentó algún nivel de depresión, con correlación significativa entre la depresión y el promedio escolar con r:-.247 y p:.009. Contribución: los resultados indican que, de manera muy débil, pero estadísticamente significativo, a mayor promedio escolar menor nivel de depresión en los alumnos. La alta frecuencia de la incidencia de la depresión en la muestra estudiada más la correlación significativa, sugieren la necesidad de implementar programas de atención a la depresión concurrente al proceso educativo.

Depresión, Promedio académico, Estudiantes universitarios

\begin{abstract}
Objective: to identify the relationship between depression and the school average in students of the Faculty of Psychology and Therapy of Human Communication, UJED. Depression was assessed by the Beck Test to 110 students. It is a quantitative, exploratory, nonexperimental, observational and cross-sectional study, with descriptive and correlational statistical analysis, with non-probabilistic sampling for convenience. Chronbach's alpha of the analyzed data was $0.89 .50 \%$ of the students presented some level of depression, with a significant correlation between depression and the school average with r: -. 247 and p: .009. Contribution: the results indicate that, in a very weak, but statistically significant way, the higher the school average, the lower the level of depression among students. The high frequency of the incidence of depression in the sample studied plus the significant correlation, suggest the need to implement depression care programs concurrent to the educational process.
\end{abstract}

Depression, Academic average, University students

Citación: HERRERA-VARGAS, Isela Vanessa, RÍOS-VALLES, José Alejandro, PÉREZ GONZÁLEZ, Ivonne y VÁZQUEZ-RÍOS, Elda Raque. Relación entre depresión y promedio escolar en estudiantes de la Facultad de Psicología y Terapia de la Comunicación Humana, UJED, campus Durango. Revista de Pedagogía Crítica. 2019, 3-10: 16-26

\footnotetext{
* Correspondencia del Autor (alexriva@ hotmail.com)

$\dagger$ Investigador contribuyendo como primer autor.
} 


\section{Introducción}

Durante los últimos años la depresión ha sido considerada como un problema que afecta sin distinguir género, edad, nivel socioeconómico etc., a un alto porcentaje de la población, convirtiéndose así en uno de los principales motivos por los cuales las personas acuden a consulta clínica (Arrivillaga, Cortés, Goicochea Jiménez, y Lozano, 2004). Los trastornos mentales constituyen una prioridad en salud pública, dado que se encuentran entre las causas más importantes de morbilidad en Atención Primaria (Arrieta, Díaz, y González, 2014).

Investigaciones en el campo de la salud mental han fijado su atención en la población universitaria, y diversos estudios señalan que los trastornos depresivos y ansiosos en estudiantes universitarios presentan una mayor tasa en comparación con la población general (Sánchez y Cáceres, 2012). Los estudiantes de las Ciencias de la Salud son personas vulnerables a presentar rasgos depresivos, debido a que en el transcurso de su formación profesional están sometidos a exigencias, estrés, frustraciones, desadaptación, entre otros, situaciones que pueden desencadenar la depresión si no son resueltas adecuadamente (Rosas, Yampufé, López, y Sotil de Pacheco, 2011).

Estudiantes aquejados por los síntomas depresivos presentan dificultades en la concentración, en cumplir con sus obligaciones académicas y lograr obtener buenas calificaciones, por no poder poner atención en clase o estudiar; por ello, los alumnos deprimidos son más propensos a abandonar sus estudios universitarios (Rosas, et al., 2011). Las personas con depresión dejan de realizar las actividades cotidianas perdiendo motivación, lo que genera deterioro social, laboral y escolar. Dado que la persona con síntomas depresivos puede no vivenciar tristeza, sino pérdida de interés o incapacidad para concentrarse, asimilar conocimientos y disfrutar de las actividades habituales (Romero, Fernández, Huerta Arizmendi, Barradas, 2015) El identificar problemas de salud mental en población universitaria es de vital importancia para el desarrollo de su educación integral y formación como futuros profesionales, implicando un gran reto dentro de las instituciones universitarias la identificación e intervención de estas problemáticas en búsqueda de una mejora de la calidad de vida de los estudiantes.

\section{Justificación}

Los trastornos mentales afectan el sistema nervioso, alterando el comportamiento, las emociones y las funciones cognitivas tales como la memoria y la percepción, afectando además la salud física y limitando la calidad de vida de quien los padece, así como de las personas que son parte de su entorno (INCyTU, 2018). Los índices de depresión van en aumento en los distintos sectores de la sociedad, incluido el sector universitario, afectando no solo al individuo, sino también repercutiendo en el nivel educativo en la sociedad (Serrano, Rojas, y Ruggero, 2013).

Hoy en día, los investigadores que se dedican al área de la salud mental, han puesto su mirada en los estudiantes universitarios, debido a que consideran que un factor que puede determinar en gran medida su desempeño académico y social puede ser la salud de su mente, ya que las condiciones de ésta pueden verse afectadas de acuerdo al nivel de exigencias académicas y las dificultades que se presenten durante el desarrollo de su aprendizaje, las cuales, plantean situaciones de fracaso o éxito escolar, y para su añadidura, al ser acompañadas con el ritmo de estudio, la pesada carga horaria, la exigencia de los docentes, las presiones y competitividad en su mismo grupo, los desórdenes en los horarios de alimentación y para variar, la alteración de su ciclo sueñovigilia debido al estudio, pueden alterar sus condiciones de salud (Gutiérrez, et al., 2010).

Así pues, lo antes mencionado despertó el interés por identificar los niveles de depresión en alumnos de las licenciaturas de Psicología y Terapia de la Comunicación Humana de la Universidad Juárez del Estado de Durango (UJED), así como su relación con el promedio escolar, ya que de esta manera se podrá identificar si existe la necesidad de la creación y puesta en marcha de programas tanto preventivos como de intervención, orientados a la mejora de la salud mental de los alumnos universitarios.

\section{Problema}

En el año 2018 la OMS dio a conocer que aproximadamente entre un $10 \%$ y $20 \%$ de los jóvenes presentan problemas de salud mental, de los cuales, la depresión es la novena causa principal de enfermedad y discapacidad en esta población (OMS, 2018).

HERRERA-VARGAS, Isela Vanessa, RÍOS-VALLES, José Alejandro, PÉREZ GONZÁLEZ, Ivonne y VÁZQUEZ-RÍOS, Elda Raque. Relación entre depresión y promedio escolar en estudiantes de la Facultad de Psicología y Terapia de la Comunicación Humana, UJED, campus Durango. Revista de Pedagogía Crítica. 2019 
De igual manera para el año 2018, las diferentes instituciones de salud de todo el país tales como la Secretaría de Salud, IMSSord, ISSSTE, IMSSop, DIF, PEMEX, SEDENA, SEMAR, entre otras, notificaron los casos nuevos de depresión en México, y como resultado se obtuvo un total de 121053 personas con este padecimiento, de los cuales 15,702 son jóvenes de 15 a 24 años; mientras que en el estado de Durango se reportaron 4,458 casos por parte de estas instituciones de los cuales 587 de esos casos corresponden a la población de 15 a 24 años (DGE, 2018).

Así mismo, la Dirección General de Epidemiología (2018) dio a conocer que en el estado de Durango en el año 2018, la depresión se encontraba en el lugar número 14 de entre las 20 principales causas de enfermedad en este estado (DGE, 2018). Según el Sistema Nacional de Vigilancia Epidemiológica (SINAVE) en el boletín epidemiológico dio a conocer que hasta la semana 32 del año 2019, tan solo en el estado de Durango se han registrado 663 casos de depresión en hombres y 2285 casos en mujeres, dando un total de 2948 personas con esta enfermedad (SSa, 2019).

Según el H. Congreso del Estado de Durango (2018), hoy en día las discapacidades psicosociales representan un serio problema de salud pública que genera un gran costo social, afectando a personas sin distinción de edad, sexo, nivel económico y sociocultural. (H. Congreso del Estado de Durango., 2018), como ejemplo, en el caso específico de los jóvenes estudiantes, estos problemas afectan considerablemente en su funcionamiento, e incluso interfieren en sus quehaceres escolares, hasta llegar al grado de no asistir a su institución educativa (OMS, 2018).

\section{Hipótesis}

Hi: La depresión evaluada con el Test de Beck en estudiantes de las carreras de Psicología y Terapia de la Comunicación Humana (FPyTCH) de la UJED está relacionada con el promedio escolar.

Ho: La depresión evaluada con el Test de Beck en estudiantes de las carreras de Psicología y Terapia de la Comunicación Humana de la UJED no está relacionada con el promedio escolar

\section{Objetivos}

\section{Objetivo General}

Determinar la relación entre la depresión y el promedio escolar en estudiantes de la Facultad de Psicología y Terapia de la Comunicación Humana, UJED.

\section{Objetivos específicos}

- Conocer el porcentaje de alumnos que presentan depresión, según la escala del Test de Beck.

- Identificar el porcentaje de alumnos que presentan depresión leve mediante la escala del test de Beck.

- Determinar el porcentaje de alumnos que presentan depresión moderada mediante la escala del test de Beck.

- $\quad$ Establecer el porcentaje de alumnos que presentan depresión severa mediante la escala del test de Beck.

\section{Marco teórico}

\section{Antecedentes}

En un estudio realizado por Arrivillaga y colaboradores en el año 2004 donde se evaluó a 218 jóvenes de pregrado de la Pontificia Universidad Javeriana- Cali mediante El Inventario de Depresión de Beck (IDB), La Escala Autoaplicada de Depresión de Zung (EAZ) y un Apartado de Datos Complementario, donde se encontró una prevalencia significativa de depresión de un 30\% según el IDB y un 25\% según la EAZ, presentando mayor frecuencia en mujeres.

Asimismo, estos autores mencionan que las variables en las dificultades académicas de la investigación se encontraron de manera significativa en los niveles de depresión moderado y severo, concluyendo que esto se puede deber, por un lado, a eventos estresores que posiblemente sean los que desencadenan los episodios depresivos, y por otro lado, podría ser ligado factores como perdida de interés y/o disminución de las actividades diarias, relacionadas con el rendimiento académico (Arrivillaga et al., 2004). 
Franco y colaboradores (2011) evaluaron una muestra de 385 estudiantes de pregrado de una universidad pública en Santa Marta, Colombia. Los instrumentos utilizados fueron la Escala breve de Zung para depresión y parte del cuestionario VESPA para la evaluación de la percepción de rendimiento académico, de donde se obtuvo que la depresión en esta investigación fue de un $38.5 \%$ (aunque sin importancia clínica), observándose que en el género femenino se presenta más elevada a comparación del género masculino $(40.3 \%$ y 37.7 respectivamente) (Franco, Gutiérrez, y Perea, 2011).

Asimismo, los resultados arrojaron una fuerte asociación entre la depresión presente los últimos quince días y la percepción de regular o mal rendimiento académico durante el último mes en los estudiantes evaluados, dando a conocer también que la depresión mayor en este estudio se presentó más elevada en mujeres que en hombres (Franco et al., 2011).

En un estudio realizado por Serrano y colaboradores en el año 2013 donde se evaluó mediante el Inventory of Depression and Anxiety Symptoms (IDAS) a 218 estudiantes de las licenciaturas de Psicología, Educación y Trabajo Social de la Universidad Autónoma del Estado de México, donde se encontró una correlación significativa entre depresión y rendimiento académico, considerándola ya sea como un causa o como un efecto de los aspectos académicos (Serrano, Rojas, y Ruggero, 2013).

En lo que respecta a México, en el año 2013 Fouilloux y colaboradores realizaron un estudio donde se evaluó mediante el inventario de Beck para la Depresión y la Subescala de Depresión del SCL90 (symptom Check-List) en 774 estudiantes del primer año la licenciatura de médico cirujano que estaban asociados con bajo rendimiento académico, y como resultado obtuvieron un porcentaje de $23 \%$ de estudiantes con síntomas depresivos (12.3\% hombres y $28.4 \%$ mujeres), asimismo dieron a conocer que el $68 \%$ que tenían síntomas depresivos reprobaron alguna materia, suponiendo un riesgo mayor de reprobación de 2.4 veces, en comparación con quienes no presentaban dicha sintomatología. Finalmente, concluyeron que la sintomatología depresiva es un factor de riesgo para la reprobación de materias o tener un bajo rendimiento escolar (Fouilloux et al, 2013).
González-Olaya y colaboradores en el año 2014 realizaron un estudio en 216 estudiantes de medicina de Colombia, de donde concluyeron que existe una alta frecuencia de síntomas depresivos de importancia clínica en estos estudiantes, presentándose mayormente en mujeres; asimismo, encontraron correlación entre dichas variables psicológicas y el resultado académico (González, Delgado, Escobar, y Cárdenas, 2014).

En un estudio realizado por Martínez y colaboradores (2016) concluyen que el rendimiento académico se encuentra ligado con el estado emocional de los estudiantes, debido a que al verse alterado este estado, no se tiene tienen las condiciones óptimas para lograr adquirir nuevos aprendizajes (Martínez, Muñoz, Rojas, y Sánchez, 2016).

En el año 2018 Moreira de Sousa y colaboradores realizaron un estudio mediante una encuesta sociodemográfica y la Escala de Ansiedad y Depresión del Hospital (HADS) en 750 universitarios portugueses, en el cual se obtuvo una prevalencia de $3.7 \%$ para los síntomas depresivos. De igual manera encontraron que los síntomas depresivos se asociaron ligeramente con un bajo rendimiento académico (Moreira de Sousa, Moreira, y Telles, 2018).

\section{Depresión}

La Organización Mundial de la Salud (OMS), define la depresión como un trastorno mental, donde la persona que lo padece presenta tristeza, pérdida de interés o placer, sentimientos de culpa o falta de autoestima, trastornos del sueño o del apetito, sensación de cansancio y falta de concentración (OMS, 2017).

Es una enfermedad que puede volverse crónica y disminuir la capacidad de las personas de trabajar, estudiar, socializar o ejecutar algunas de sus actividades cotidianas; dependiendo del número y de la intensidad de los síntomas, los episodios depresivos pueden clasificarse como leves, moderados o graves. Las personas que sufren de depresión grave necesitan tratamiento; ellas pueden pensar, intentar o lograr suicidarse. Si la depresión es leve, se puede tratar sin necesidad de medicamentos (OMS, 2017). 
La depresión, en su sentido más común y más popular, es un síntoma anímico, aunque también puede ser un síndrome, es decir, un conjunto de síntomas aunados al del bajo estado de ánimo; los típicos síntomas acompañantes son insomnio, falta de apetito, pérdida de peso, etcétera; y en su sentido más restrictivo, "lo que entendemos como trastorno depresivo no es sólo un síndrome depresivo sino un cuadro clínico completo con una duración determinada, con un patrón de síntomas exigibles para su diagnóstico" (Ortiz, 1997: 142, citado en, Serrano, Rojas y Ruggero, 2013, pp. 47-60).

La premisa básica de la teoría de Beck es que la depresión se caracteriza fundamentalmente por la existencia de errores cognitivos que conducen al individuo deprimido a percibir e interpretar la realidad de una manera negativa. Es decir, en términos del paradigma del procesamiento de información, la depresión se caracterizaría por un procesamiento distorsionado negativamente de la información (Ortíz, 1997).

Actualmente se ha señalado que la depresión es el resultado de la interacción entre varios factores, de los cuales se pueden mencionar a los factores biológicos, heredados, del desarrollo, y del contexto, los cuales interactúan con las características de acuerdo a cada individuo. Es por ello, que su estudio requiere de diversas aproximaciones, reconociendo la importancia del análisis de los avances en el campo de las neurociencias y la genética, las cuales han dotado las bases para la corrección de la dualidad mente-cuerpo, y que constituyo una barrera para el entendimiento de la relación entre el cerebro, el pensamiento, la emoción y la conducta (Berenzon, Lara, Robles, y Medina, 2013).

La depresión no se distingue de una edad en específica, es decir, puede ocurrir a cualquier edad, pero a menudo comienza en la etapa de la adolescencia o a principios de la vida adulta temprana, es decir, aproximadamente entre los 20 y los 35 años de edad (NIH, 2015).

Según el National Institute of Mental Health (2015) existen varios tipos de depresión los cuales a continuación se describirán:
Depresión grave: se presentan síntomas graves que interfieren con el trabajo, el sueño, el estudio, la comida y el disfrutar de la vida. Probablemente una persona solo tenga un episodio de depresión grave en la vida, pero es más común tener varios de esos episodios.

Trastorno depresivo persistente: es un estado de ánimo depresivo que tiene una duración de por lo menos 2 años. La persona que es diagnosticada con trastorno depresivo persistente puede tener también episodios de depresión grave, junto con periodos de síntomas menos graves.

También pueden existir otras formas de depresión que son ligeramente diferentes o pueden presentarse bajo circunstancias únicas, estas son:

Depresión psicótica: esta se produce cuando una persona tiene depresión grave, más algún tipo de psicosis, como creencias falsas que le perturban, delirios o alucinaciones.

Depresión afectivo estacional: se caracteriza por la aparición de depresión durante los meses de invierno o cuando hay menos luz solar natural. Este tipo de depresión generalmente suele desaparecer durante la primavera y el verano.

Trastorno bipolar: esto es diferente a la depresión, aunque la razón por la que aparece en esta lista es porque las personas con trastorno bipolar tienen episodios de ánimo extremadamente bajos (o depresión) o pueden tener estados de ánimo extremadamente elevados (llamados manía) (NIH, 2015).

\section{Desempeño académico}

Edel Navarro (2003) define el rendimiento académico como "un constructo susceptible de adoptar valores cuantitativos y cualitativos, a través de los cuales existe una aproximación a la evidencia y dimensión del perfil de habilidades, conocimientos, actitudes y valores desarrollados por el alumno en el proceso de enseñanza aprendizaje" (Edel, 2003). Uno de los objetivos del rendimiento escolar o académico es lograr el alcance de un aprendizaje, y sus componentes son procesos que se promueven principalmente en la escuela, los cuales consisten en la transformación de un determinado estado en otro completamente nuevo (Lamas, 2015). 
Las calificaciones escolares son una de las variables que los docentes e investigadores más utilizan o toman en consideración para aproximarse al rendimiento académico (Edel, 2003). Durante los años setentas y ochentas, y aun en la actualidad, las calificaciones son consideradas como un criterio de rendimiento académico (Lamas, 2015).

Morales y colaboradores (2016) no consideran al rendimiento escolar como una variable neutra, ya que en sus palabras, éste produce efectos tanto positivos como negativos, es decir, cuando los estudiantes alcanzan evaluaciones que los acreditan en su aprendizaje, se crea en ellos autoconfianza, motivación y autoestima que se puede ver reflejada en su comportamiento; y, por el otro lado, al obtener resultados negativos y no lograr la acreditación de su aprendizaje, se crean en ellos pensamientos tales como "no sé", "no puedo", "no sirvo" y hasta "no nací para estudiar", y por ende, al desconfiar de sus habilidades y conocimiento, esto los va llevando a tener una baja autoestima, un bajo rendimiento académico y en casos extremos hasta la deserción escolar (Morales, Morales, y Holguín, 2016).

\section{La depresión y el desempeño académico}

Borges (2016) menciona que algunos síntomas de la depresión que perjudican el desempeño escolar son la falta de iniciativa y la baja autoestima, y además síntomas, al entrar en combinación unos con otros, pueden interferir a nivel global en el estudiante, manifestándose en el ambiente escolar, considerando que algunas habilidades tales como la memoria, la concentración, la atención y otras más, juegan un papel esencial para el aprendizaje (Borges, 2016). Algunos estudios han señalado que el bajo desempeño escolar se presentó más frecuentemente en alumnos deprimidos, asociando a la depresión con dificultades de concentración, relaciones sociales y desempeño en la lectura y la escritura, incluso, percibían la tarea como una carga pesada (Borges, 2016).

Así mismo, Roca y colaboradores (2015), mencionan que los síntomas cognitivos, derivados de alteraciones en alguna de las funciones cognitivas, constituyen una característica central y nuclear de los trastornos afectivos depresivos. El rendimiento académico en estudiantes universitarios ha sido relacionado con un amplio número de factores (Roca, Vives, Navarro, García, y Gili, 2015).

ISSN 2523-2479

ECORFAN $^{\circledR}$ Todos los derechos reservados
De igual manera manifiestan que un estudiante con mal rendimiento académico puede generar variados problemas, tales como la insatisfacción personal, falta de motivación, hasta una baja autoestima, entre otros (Roca et al., 2015).

\section{Test de depresión de Beck (BDI-IA)}

La escala de depresión de Beck, es uno de los instrumentos que se usan frecuentemente para evaluar los síntomas depresivos (González et al., 2014).

El inventario de Depresión de Beck, segunda edición (BDI-IA) es un instrumento de autoinforme compuesto por 21 ítems, cuya finalidad es medir la severidad de la depresión en adultos y adolescentes a partir de los 13 años. Esta versión del BDI fue desarrollada para evaluar los síntomas correspondientes a los criterios diagnósticos de los trastornos depresivos descriptivos en el Manual Diagnóstico y Estadístico de los Trastornos Mentales Cuarta Edición (DSM-IV, 1994) de la American Psychiatric Asociation (Beck, 2009).

Después de 35 años de investigación y experiencia con el BDI, los mismos autores consideraron necesario revisar y modificar el Beck Depression Inventory (BDI-IA; Beck, Rush, Shaw y Emery, 1979) y modernizarlo (Beck, 2009).

El BDI-IA reemplazó al instrumento original (BDI) que había sido desarrollado por Beck, Ward, Mendelson, Mock y Erbaugh (1961). En la nueva versión que aquí se presentaBDI-II-cuatro ítems (Pérdida de Peso, Cambio de Imagen Corporal, Preocupación Somática y Dificultad para Trabajar) fueron reemplazados por otros nuevos (Agitación, Desvalorización, Dificultad de Concentración y Pérdida de Energía) con el objetivo de identificar síntomas típicos de la depresión que requiere hospitalización. Se cambiaron dos ítems para incluir los cambios en el apetito y en el sueño (tanto los aumentos como las disminuciones). Además, se reformularon algunos enunciados (o alternativas) utilizados para evaluar los otros síntomas. A diferencia del BDI-IA, el BDI-II constituye una revisión sustantiva del BDI original (Beck, 2009). 
Los resultados obtenidos indican que el Inventario de Beck reúne los requisitos psicométricos para su utilización en forma válida en nuestro medio. En cuanto a composición factorial, validez de constructo, validez discriminante y consistencia interna, corroboraron las adecuadas propiedades psicométricas del BDI-IA, las cuales lo avalan como un instrumento suficientemente válido y confiable para la medición de los síntomas depresivos en población mexicana, con lo cual también apoya la validez transcultural del instrumento (Beck, 2009); (Beltrán, 2012).

Estudios recientes del BDI-IA han arrojado una estructura factorial de dos dimensiones, en concordancia con el modelo original propuesto por Beck, Steer \& Garbin (1988). El factor 1 se denomina cognitivoafectivo y el factor 2, somático-vegetativo, los cuales explican el $37.41 \%$ y $6.28 \%$ de la varianza respectivamente, y muestran un alfa de Cronbach de .89 para el primero y .72 para el segundo (Mukhtar \& Tian, 2008). No obstante, Steer, Beck y Brown (1989) han advertido que este arreglo podría variar de una población clínica a otra, lo que hace necesaria su confirmación en otras poblaciones (Beltrán, 2012).

Así mismo, los puntajes de corte propuestos por Beck et al. (1996) resultaron apropiados para discriminar los distintos grados de depresión (ver tabla 1).

\begin{tabular}{|l|l|}
\hline $\begin{array}{l}\text { Puntuaciones totales } \\
<=9\end{array}$ & \multicolumn{1}{|c|}{ Nivel de depresión } \\
\hline $10-15$ & Lormal \\
\hline $16-24$ & Moderadamente deprimidos \\
\hline $25-62$ & Gravemente deprimidos \\
\hline
\end{tabular}

Tabla 1 Puntaje total para la interpretación de los resultados del inventario del Test de Beck

\section{Metodología de investigación}

El presente estudio es de tipo exploratorio, no experimental, observacional y transversal, con un análisis estadístico descriptivo y correlacional. Se analizó la variable compleja depresión y para categorizar el desempeño académico se utilizó el promedio escolar (referido por los alumnos), además se consideraron las variables signalíticas de: edad y género.
Para el análisis estadístico de las características de la población estudiada se emplearon medidas de tendencia central (media, moda, mediana) y de dispersión (desviación estándar) para variables cuantitativas, para las variables cualitativas se utilizaron frecuencias y proporciones y para el análisis correlacional se empleó el coeficiente de Pearson, dada la prueba de bondad de ajuste.

Como criterios de inclusión se consideraron a alumnos de $2^{\circ}$ a $8^{\circ}$ semestre de las carreras de Psicología y Terapia de la Comunicación Humana de la UJED, que desearan participar en el estudio y hayan firmado la carta de consentimiento informado, como criterio de eliminación se descartaron aquellos alumnos que decidieran retirar su consentimiento informado en cualquier momento de la evaluación, aquellos que tuvieran incompleta la información y a los alumnos que refirieran alguna alteración psiquiátrica y/o psicológica; debido a este criterio se eliminaron 8 casos, quedando un total de 110 casos válidos.

\section{Procedimiento}

Se invitó a los alumnos de las carreras de Psicología y Terapia de la Comunicación Humana de la FPyTCH UJED, de $2^{\circ}$ a $8^{\circ}$ semestre. La toma de datos se realizó durante el periodo del mes de mayo del 2019, culminando en julio del mismo año.

Previa firma del consentimiento informado de acuerdo con las normas oficiales mexicanas 004-ssa3-2012 del expediente clínico y 040-ssa2-2004 en materia de información, se procedió a la aplicación de los instrumentos para la recolección de datos e interpretación de los resultados.

Para el estudio del nivel de depresión en los alumnos se utilizó el test de depresión de Beck, el cual es un inventario autoaplicado de 21 ítems que evalúa la intensidad de la depresión. En cada uno de los ítems el sujeto tiene que elegir la frase que, entre un conjunto de cuatro alternativas (siempre ordenadas por su gravedad) se ajusta mejor a su estado de ánimo durante la última semana. Cada ítem se valora de 0 a 3 puntos en función de la alternativa escogida, la puntuación total resultante tiene un rango entre 0 y 63 puntos. El tiempo de duración en la aplicación del Test, va de 10 a 15 minutos aproximadamente. 
La identificación de las variables signalíticas y el rendimiento académico (género, edad y promedio escolar) se obtuvo mediante la aplicación de una encuesta clínica.

El análisis estadístico de la información obtenida, se realizó mediante el programa Statistical Package for the Social Sciences (SPSS) versión 20.

\section{Resultados}

La muestra estudiada estuvo conformada por 110 participantes, teniendo 31 alumnos $(28.2 \%)$ pertenecientes a la carrera de Psicología y 79 alumnos $(71.8 \%)$ de la carrera de Terapia de la Comunicación Humana, de los cuales 96 (87.3\%) corresponden a individuos del género femenino y 14 (12.7\%) del género masculino (Ver tabla 2 y 3 ).

\begin{tabular}{|l|r|r|}
\hline Licenciatura & Total alumnos & Porcentaje \\
\hline TCH & 79 & $71.8 \%$ \\
\hline Psicología & 31 & $28.2 \%$ \\
\hline
\end{tabular}

Tabla 2 Frecuencia de alumnos por licenciatura de la Facultad de Psicología y Terapia de la Comunicación Humana

\begin{tabular}{|l|r|r|}
\hline Género & \multicolumn{1}{r|}{ Total } & Porcentaje \\
\hline Femenino & 96 & $87.3 \%$ \\
\hline Masculino & 14 & $12.7 \%$ \\
\hline
\end{tabular}

Tabla 3 Frecuencia por género

La edad de los participantes estuvo en un rango de 18 a 30 años. La media de la edad fue de 20.8 años, con una moda de 20 años, la desviación estándar fue de 1.79 .

La confiabilidad de los resultados obtenidos en la evaluación del Test de depresión de Beck de los alumnos de Psicología y Terapia de la Comunicación Humana fue de 0.89.

El promedio escolar referido por los participantes de la muestra estudiada presentó puntajes con un valor mínimo de 7.0 y un valor máximo de 9.8 , con una media de 8.8 , una moda y mediana de 8.9 , desviación estándar de 0.50 (ver gráfica 1).

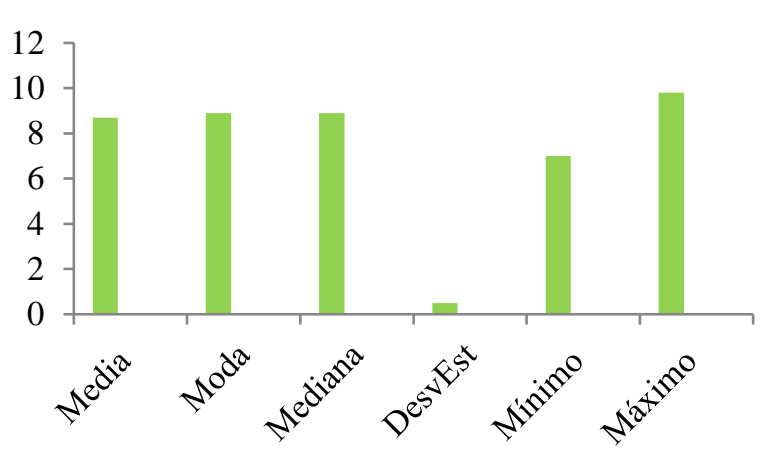

Gráfica 1 Medidas de tendencia central para el promedio escolar

En cuanto a los resultados obtenidos de la evaluación de la depresión mediante el Test de Beck se obtuvo que 55 alumnos (50\%) no muestran ningún nivel de depresión, mientras que el otro $50 \%$ muestra algún grado de depresión (Ver tabla 4).

\begin{tabular}{|c|c|c|c|}
\hline Rango & Frecuencia & Porcentaje & $\begin{array}{l}\text { Nivel de } \\
\text { depresión }\end{array}$ \\
\hline$<=9$ & 55 & $50 \%$ & normal \\
\hline $10-15$ & 21 & $19.1 \%$ & Leve \\
\hline 16-24 & 22 & $20 \%$ & Moderado \\
\hline $25+$ & 12 & $10.9 \%$ & Severo \\
\hline
\end{tabular}

Tabla 4 Resultados de la evaluación del Test de Beck

La media de la puntuación de Beck fue de 11.6, con desviación estándar de 9.4, un valor mínimo de 0 y máximo de 46 (Ver gráfica 2).

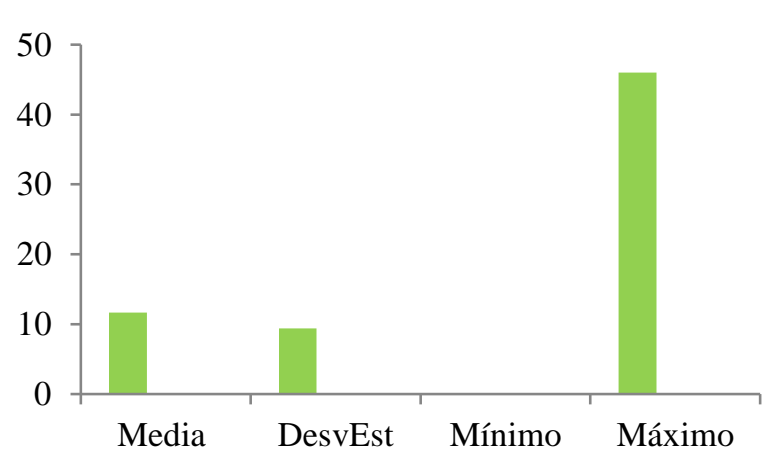

Gráfica 2 Medidas de tendencia central del puntaje total de Beck

Así mismo, la media del puntaje de los alumnos que presentan depresión es de 19, con desviación estándar de 7.9, lo cual indica que se ubican en un nivel de depresión moderado. La media de los alumnos que no presentan depresión es de 4.36, con desviación estándar de 2.89 . 
La media del promedio escolar de los alumnos que presentan depresión es de 8.6, con desviación estándar de 0.49 , mientras que la media del promedio escolar de los alumnos que no presentan depresión es de 8.9, con desviación estándar de 0.51 (Ver gráfica 3).

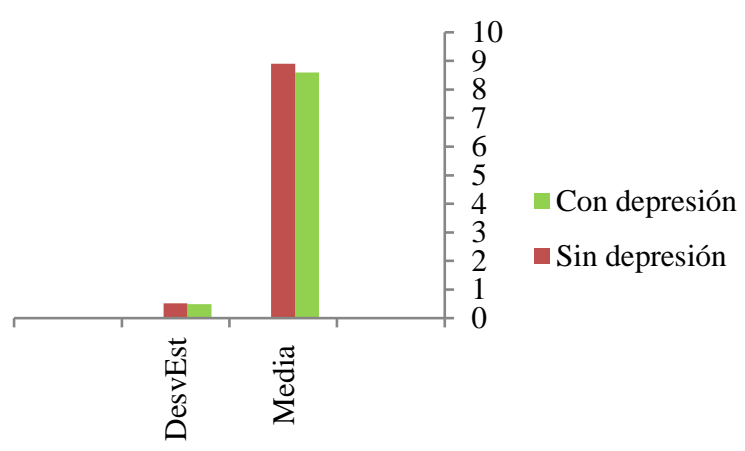

Gráfica 3 Media y Desviación estándar de alumnos con depresión y alumnos sin depresión

\section{Análisis correlacional}

Al realizar un análisis correlacional de Pearson, del promedio escolar con el puntaje del nivel de depresión se encontró una significancia negativa débil con una r-.247, y p.009, con una confiabilidad al 99\%, lo cual indica que, de manera débil, a mayor promedio escolar menor será el nivel de depresión que muestren los alumnos.

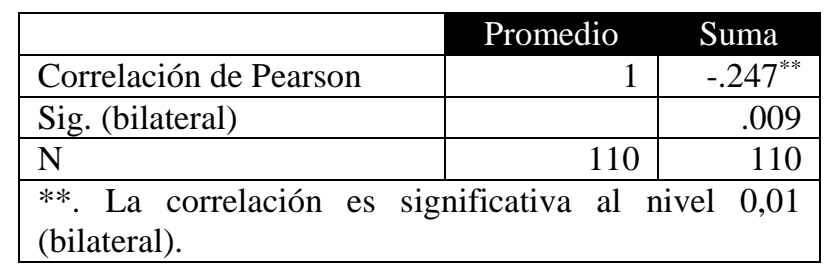

\section{Conclusión}

Tras haber realizado un análisis descriptivo y correlacional de los datos recabados en cuanto a los niveles de depresión y promedio escolar, se concluye lo siguiente:

Los resultados obtenidos de la evaluación de la depresión arrojaron que la mitad de la población estudiada (55 participantes) presenta algún nivel de depresión, de los cuales el $19.1 \%$ se ubica en un nivel de depresión leve, el $20 \%$ moderada y el $10.9 \%$, severa; identificando que de este 50\%, 43 (39.1\%) alumnos se sitúan entre los niveles de depresión leve y moderada, teniendo a 12 alumnos en un nivel de severidad.
La media del total del Test de Beck fue de 19, con una desviación estándar de 9.4, evidenciando que los alumnos que presentan depresión, se centran en el nivel moderado. Los datos obtenidos en la presente investigación concuerdan con el estudio realizado por Arrivillaga y colaboradores (2004), donde se evaluó a jóvenes de pregrado con el Test de Beck, encontrando una prevalencia significativa de depresión de un $30 \%$, identificando también a esta población en los niveles de depresión moderada.

En cuanto a los resultados de la correlación entre depresión y el desempeño académico se encontró relación significativa débil con un nivel de confiabilidad al $99 \%$, aceptando así la hipótesis alterna. Así mismo, estos resultados concuerdan con el estudio realizado por Serrano y colaboradores en el año 2013, donde evaluaron alumnos universitarios de la Universidad Autónoma del Estado de México, donde se encontró una correlación significativa entre depresión y rendimiento académico. La alta frecuencia de la incidencia de la depresión en la muestra estudiada más la correlación significativa, sugieren la necesidad de implementar programas de atención a la depresión concurrente al proceso educativo.

Ya con lo anterior expuesto, sería recomendable que en las Instituciones de educación Superior, los docentes se motiven e involucren en la prevención y detección temprana de posibles síntomas depresivos en sus estudiantes. La identificación temprana de la depresión no solo minimizaría la posibilidad de un bajo rendimiento académico, sino que también reduciría otras conductas de riesgo para la salud, con la presencia de trastornos depresivos (Campo, Díaz, Rueda, y Barros, 2005).

\section{Agradecimiento}

Un sincero agradecimiento a la Facultad de Psicología y Terapia de la Comunicación Humana, por medio de su directora la MTF. Elda Raquel Vázquez Ríos, por las facilidades otorgadas para que se llevara a cabo el presente trabajo de investigación, al Dr. José Alejandro Ríos Valles, pionero de este proyecto, así como a los alumnos que decidieron participar en el estudio. Gracias por el apoyo brindado. 


\section{Referencias}

Arrieta, K., Díaz, S., \& González, F. (2014). Síntomas de depresión y ansiedad en jóvenes universitarios: prevalencia y factores relacionados. REV CLIN MED FAM., 14-22.

Arrivillaga Quintero, M., Cortés García, C., Goicochea Jiménez, V. L., \& Lozano Ortíz, T. M. (2004). Caracterización de la depresión en jóvenes universitarios. Universitas Psychologica, 3(1), 17-26.

Beck, A. T. (2009). Inventario de Depresión de Beck: Segunda edición (1a ed.). Buenos Aires.: Paidós, 2009.

Beltrán, M. d.-Á.-G. (2012). El Inventario de Depresión de Beck: Su validez en población adolescente. Terapia psicológica., 30(1), 5-13.

Berenzon, S., Lara, M., Robles, R., \& MedinaMora, M. (2013). Depresión: estado del conocimiento y la necesidad de políticas públicas y planes de acción en México. Salud Pública Méx, 55(1), 74-80.

Borges, L. (2016). Sintomatología depresiva y desempeño escolar: un estudio con niños brasileños. Ciencias Psicológicas, 10(2), 189197.

Campo, A., Díaz, L., Rueda, G., \& Barros, J. (2005). Validación de la escala de Zung para depresión en universitarios de Bucaramanga, Colombia. Revista colombiana de Psiquiatría, 54-62.

Dirección General de Epidemiología . (2018). Anuario de Morbilidad 1984-2018. México : Gobierno de México .

Edel Navarro, R. (2003). El rendimiento académico: concepto, investigación y desarrollo. Revista Iberoamericana sobre Calidad, Eficacia y Cambio en Educación, 1(2).

Fouilloux Morales, C., Barragán Pérez, V., León, O., Silvia, Jaimes Medrano, A., Urrutia Aguilar, M. E., \& Guevara-Guzmán, R. (2013). Síntomas depresivosy rendimiento escolar en estudiantes de Medicina. Salud mental, 36(1), 59-65.
Franco Mejía, C., Gutiérrez Agudelo, S., \& Perea, E. (2011). Asociación entre depresión y bajo rendimiento académico en estudiantes universitarios. Psicogente, 14(25), 67-75.

González-Olaya, H. L., Delgado-Rico, H. D., Escobar-Sánchez, M., \& Cárdenas-Angelone, M. E. (2014). Asociación entre el estrés, el riesgo de depresión y el rendimiento académico entre estudiantes de los primeros semestres de un programa colombiano de medicina. Revista de la Fundación Educación Médica, 17(1), 47-54.

Gutiérrez Rodas, J. A., Montoya Vélez, L. P., Toro Isaza, B. E., Briñón Zapata, M. A., Rosas Restrepo, E., \& Salazar Quintero, L. E. (2010). Depresión en estudiantes universitarios y su asociación con el estrés académico. CES Medicina, 24(1), 7-17.

H. Congreso del Estado de Durango. (2018). Gaceta parlamentaria. Durango: Legislatura Durango.

INCyTU. (2018). Salud mental en México . Ciudad de México: Oficina de Información Científica y Tecnológica para el Congreso de la Unión .

Lamas, H. A. (2015). Sobre el rendimiento escolar. School performans. Propósitos y representación, 3(1), 313-386.

Martínez-Martínez, M. d., Muñoz-Zurita, G., Rojas-Valderrama, K., \& Sánchez-Hernández, J. A. (2016). Prevalencia de síntomas depresivos en estudiantes de la Licenciatura de medicina de Puebla, México. ScienceDirect, 23(4), 145-149. doi:https://doi.org/10.1016/j.af.2016.10.004

Morales Sánchez, L. A., Morales Sánchez, V., \& Holguín Quiñonez, S. (2016). Rendimiento escolar. Humanidades, Tecnología y Ciencia del Instituto Politécnico Nacional(15), 1-5.

Moreira de Sousa, J., Moreira, C., \& TellesCorreia, D. (2018). Anxiety, Depression and Academic Performance: A Study Amongst Portuguese Medical Students Versus NonMedical Students. Act Med Port, 31(9), 454-462. doi:10.20344/amp.9996

Mukhtar, F., \&Tian, O. (2008).Exploratory and confirmatory factor validation and psychometric properties of the Beck Depression Inventory for Malays (BDI-Malays) in Malaysia.Malaysian Journal of Psychiatry, 17, 17-25

HERRERA-VARGAS, Isela Vanessa, RÍOS-VALLES, José Alejandro, PÉREZ GONZÁLEZ, Ivonne y VÁZQUEZ-RÍOS, Elda Raque. Relación entre depresión y promedio escolar en estudiantes de la Facultad de Psicología y Terapia de la Comunicación Humana, UJED, campus Durango. Revista de Pedagogía Crítica. 2019 
National Institute of Mental Health. (2015). La depresión: lo que usted debe saber. Bethesda: $\mathrm{NIH}$.

OMS . (2018). Salud mental en el adolescente . Ginebra : Organización Mundial de la Salud.

Organización Mundial de la Salud. (7 de abril de 2017). Temas de salud: Depresión . (OMS, Ed.) Recuperado el 23 de julio de 2018, de Temas de salud: Depresión

https://www.who.int/topics/depression/es/

Ortíz Tallo, M. (1997). Trastornos Psicológicos (1a Edición/ Rústica/ Castellano ed.). Madrid, España: Ediciones Aljibe, S.L.

Roca, M., Vives, M., Navarro, E., GarcíaCampayo, J., \& Gili, M. (2015). Alteraciones cognitivas y depresión: una revisión crítica. Actas Esp Psiquiatr, 43(núm 5), 187-193.

Romero Ascaino, M. E., Fernández Mojica, N., Huerta Arizmendi, G., \& Barradas Alarcón, M. E. (2015). Depresión y ansiedad en estudiantes de pedagogía Veracruz, Universidad Veracruzana. RIDE Revista Iberoamericana para la Investigación y el Desarrollo Educativo., 6(11), 1-12.

Rosas, M., Yampufé, M., López, M., \& Sotil de Pacgheco, A. (2011). Niveles de depresión en estudiantes de Tecnología Médica. Anales de la Facultad de Medicina, Vol. 72(3), 183-186.

Sánchez, R., \& Cáceres, H. (2012). Ideación suicida en adolescentes universitarios: Incidencia y factores asociados. . Biomedica., 716.

Secretaría de Salud. (2019). Boletín epidemiológico. Sistema Nacional de Vigilancia Epidemiológica. Sistema Único de Información. México : Secretaría de Salud . Recuperado el 22 de Agosto de 2019

Serrano Barquín, C., Rojas García, A., \& Ruggero, C. (2013). Depresión, ansiedad y rendimiento académicon en estudiantes universitarios. Revista Intercontinental de Psicología y Educación, 15(1), 47-60. 